\title{
Driver behaviour models for a driving simulator-based intelligent speed adaptation system
}

\author{
S. A. $\operatorname{Arhin}^{1} \&$ A. Eskandarian ${ }^{2}$ \\ ${ }^{1}$ Howard University, USA \\ ${ }^{2}$ George Washington University, USA
}

\begin{abstract}
Intelligent Speed Adaptation systems (ISAs) have been evaluated in both simulator and field operation experiments largely in Europe, and most recently in the United States, for their efficacy in mitigating excessive speeding. In most of the simulator experiments, varying roadway scenarios have been used to mimic real-life driving conditions. In these experiments, with the introduction of ISAs in virtual driving scenarios, the deviation from reality may even be compounded. In this paper, regression models for three types of ISAs, namely, Warning, Mandatory and the Advanced Vehicle Speed Adaptation System (AVSAS), which predict driving behaviour on approach to a stop-controlled intersection and a curve, are presented. On approach to the stop-sign, the stopping distance was predicted with the maximum deceleration rate, approach speed and deceleration time as the regressors. On curve approaches, the approach speed of the drivers was predicted based on the maximum deceleration rate and deceleration time. The regression models had associated $R^{2}$ values ranging from $66 \%$ to $93 \%$. These models could be used to enhance or refine the realism of the roadway designs for simulator-based ISA experiments. In addition, these models, when further validated in field operational tests, can be incorporated in the development of future ISA algorithms as predictors of driver behaviour.
\end{abstract}

Keywords: intelligent speed adaptation systems, deceleration, braking.

\section{Introduction}

A considerable number of studies have been conducted on the prediction of driver behaviour on different highway segments in the last decade. These models 
have largely been developed to predict the speeding behaviours using the $85^{\text {th }}$ percentile or average speeds of a population of drivers for a number of highway facilities. In most simulator experiments, researchers rely on pre-designed roadway simulator graphics and systems with design elements that may not adequately reflect the real world driving scenarios. Thus, road segments in these experiments are often simulator-based designs, with some references to the design standards in American Association of State Highway and Transportation Officials (AASHTO) as well as the Manual on Uniform Traffic Control Devices (MUTCD). In addition, since most driving simulators are deficient in one way or another in mimicking real driving conditions, having models which deal with specific roadway scenarios for a simulator would be useful in the prediction of driver behaviour in a fixed-based simulator. Some of the driver behaviours which could be influenced in a fixed-based simulator include speed, stopping distance and deceleration rate.

Intelligent Speed Adaptation systems (ISAs) are in-vehicle systems that have the capability of either warning drivers of adverse speeding behaviours or limit them from exceeding a prevailing speed limit or advisory. A good majority of ISAs have been tested using driving simulators. The application of driver behaviour models in the design and development of ISA systems would result in a more effective system since they would incorporate driver behaviour under different scenarios. Most ISA systems developed to date have not relied on such models. In this paper, two types of regression models for predicting driving behaviour using a driving simulator, augmented with additional systems, are presented. Using simple regression analysis methods, two models for general situations were developed:

- Stopping distance at stop-controlled intersections, and

- Approach speed on curves

These models were developed based on data gathered during the conduct of an experiment to compare three ISAs in a driving simulator. The scenarios for the simulator experiment are briefly described and referred in this paper for the three ISAs: 1) Warning, 2) Mandatory, and 3) AVSAS. The Warning ISA provides alarms and notifications when the system identifies and determines that a potentially hazardous situation is about to occur that requires immediate driver action. The Mandatory ISA system controls the maximum speed that can be attained with the driver not having the capability to override the system. Finally, the AVSAS technology was designed to limit the driver to a tolerable speed above the posted speed limit beyond which the driver will have to override the system. The driver behaviour models developed for unsignalized intersections and curve approaches in this study are geared toward potentially enhancing the simulator fidelity as well as improve the highway design process for ISA tests in a fixed-based simulator.

\section{Literature review}

Modelling of deceleration rates associated with speed up manoeuvres and speed change cycles is essential for the analysis of fuel consumption, operating cost, 
and pollutant emissions, as well as for determining geometric, stopped and queuing components of overall delay [1]. In addition, the modelling of deceleration characteristics and behaviour of individual drivers is key issue in the development of traffic simulation models.

Research conducted on deceleration rates in 1960 [2] assumed that a deceleration rate of $16.4 \mathrm{ft} / \mathrm{s}^{2}\left(5 \mathrm{~m} / \mathrm{s}^{2}\right)$ would be required to bring a vehicle to a stop while in another study [3], a $9.8 \mathrm{ft} / \mathrm{s}^{2}\left(2.93 \mathrm{~m} / \mathrm{s}^{2}\right)$ deceleration rate was judged to be more realistic. From additional studies and publications in the years following $[4,5]$, reasonable deceleration rates have been recommended to be between $6.9 \mathrm{ft} / \mathrm{s}^{2}\left(2.1 \mathrm{~m} / \mathrm{s}^{2}\right)$ and $13.8 \mathrm{ft} / \mathrm{s}^{2}\left(4.2 \mathrm{~m} / \mathrm{s}^{2}\right)$, depending on the mean approach speed. It has also been recommended by the 2001 AASHTO A policy on Geometric Design of Highways and Streets [1] that a deceleration rate of 11.2 $\mathrm{ft} / \mathrm{s}^{2}\left(3.41 \mathrm{~m} / \mathrm{s}^{2}\right)$ is typically comfortable for most drivers. These recommended deceleration rates assume that most drivers brake uniformly on approach to a road hazard or a regulated stop. Other studies [2,6] have shown that drivers decelerate non-uniformly and that the deceleration rates are largely dependent to the approach speed.

In a study [7] conducted in 1978 on 17 passenger cars, a constant deceleration rate model was developed which assumed that the drivers maintained an average deceleration rate throughout the time deceleration took place, in contrast to other mentioned studies. The model predicts the vehicle speed based on a linear relationship with the deceleration time. Another study [8] showed a polynomial for the vehicle speed for vehicles at higher speeds. The polynomial was of the form:

where

$$
\mathrm{v}=\mathrm{v}_{0}-\mathrm{a}_{0} \mathrm{v}_{0} \mathrm{t}^{2}
$$

$\mathrm{v}=$ vehicle speed $(\mathrm{km} / \mathrm{h})$

$\mathrm{v}_{0}=$ vehicle approach speed $(\mathrm{km} / \mathrm{h})$

$\mathrm{t}=$ deceleration time $(\mathrm{s})$ and

$\mathrm{a}_{0}=$ model constant.

From this model, it was inferred that the deceleration rate, which is the first derivative of equation (1), is directly proportional to the approach speed. Thus, the higher the approach speeds, the higher the deceleration rates. Another polynomial model [1] recommended for use in practice implied that drivers generally decelerate over a longer distance especially at higher speeds.

Two deceleration rate models for curves on two-lane rural roads were developed in a study conducted in 2007 [9]. In that study, which used data on 18 two-lane rural road horizontal curves, the results show that the approaching speed, curve length, and the radius are correlated to deceleration rate. The first deceleration rate model was defined as a function of impending curve radius, while the second estimates it as a function of speed reduction and approaching speed. The authors contended that the models predict deceleration rates consistent with observed speed reduction behaviour and implied they can be used effectively for operating speed-profile construction.

In a study conducted using data gathered during a 1996 Intelligent Cruise Control (ICC) Field Operational Test in Michigan, driver deceleration and acceleration behaviour at stop sign-controlled intersections on rural highways 
were evaluated [10]. For the acceleration and deceleration events in the experiment, a mathematical model was developed which predicts the average rates of deceleration and acceleration for each of the events. These average rates were compared for different independent variables and the results, according to the researchers, showed a strong and statistically significant dependence of the deceleration rate on the initial speed but little dependence on any of the other factors studied, including final speed, driver gender, and street type. The analysis also showed that there is a large and stochastic component in the rate of deceleration or acceleration which is influenced by driver choice.

The required overall safe stopping distance, D, depends on the driver's perception and reaction time, the grade of the roadway, as well as the vehicle reaction and braking capability [11]. Typically, D is computed as a sum of two distances, namely, the distance traversed by the vehicle from the instant the driver sights an object necessitating a stop to the instant when the brakes are applied $\left(D_{1}\right)$, and the distance needed to stop the vehicle from the instant brake application begins $\left(\mathrm{D}_{2}\right)$.

Generally, the first component is computed using the following expression:

$$
\mathrm{D}_{1}=1.47 \mathrm{Vt} \text {, }
$$

where

$$
\begin{aligned}
& V=\text { approach speed ( } \mathrm{mph} \text { ) } \\
& \mathrm{t}=\text { brake reaction time (seconds). }
\end{aligned}
$$

An assumed driver brake reaction time of 2.5 seconds is typically used.

The second component is defined as:

$$
\mathrm{D}_{2}=\frac{V^{2}}{30\left[\left(\frac{a}{32.2}\right) \pm G\right]}
$$

where

$$
\begin{aligned}
& \mathrm{V}=\text { approach speed }(\mathrm{mph}) \\
& \mathrm{a}=\text { deceleration rate }\left(\mathrm{ft} / \mathrm{sec}^{2}\right) \\
& \mathrm{G}=\text { percent of grade. }
\end{aligned}
$$

Typically, for normal braking, vehicle deceleration rate of $10 \mathrm{ft} / \mathrm{s}^{2}\left(3.04 \mathrm{~m} / \mathrm{s}^{2}\right)$ is used.

Thus

$$
\mathrm{D}=1.47 \mathrm{Vt}+\frac{V^{2}}{30\left[\left(\frac{a}{32.2}\right) \pm G\right]}
$$

For wet pavements, this distance is computed using the following equation:

$$
\mathrm{D}=1.47 \mathrm{Vt}+1.075 \frac{V^{2}}{a},
$$

where the symbols have the same definitions as above.

This distance is an important design parameter in that it defines the minimum distance that must be provided at critical points along the highway, particularly on approach to signalized and unsignalized intersections. Thus, it influences geometric design values, construction costs, and highway safety. This basic 
model for calculating stopping distances was formalized in 1940, and the parameters have been altered to compensate for changes in the assumed values for object height, eye height and driver behaviour over the past 50 years.

In recent studies, the model's parameters and assumptions have been thought not to be representative of real-world conditions. New models for determining stopping distance have been developed for geometric design of highways. In a recent study [12], which involved 50 drivers, 3,000 braking manoeuvres, 1,000 driver eye heights, and 1,000 accident narratives, a model was developed with parameters describing driver and vehicle capabilities that can be validated with field data. The model resulted in an adequate stopping distance, sag vertical curve length, and lateral clearance formulations which are between the current AASHTO-recommended minimum and desirable requirements.

In another study [13], it was contended that the existing stopping distance models are based on daytime driving and thereby rendering the model's output unrealistic for nighttime driving. The analysis of the stopping distance data collected from nighttime driving experiment show that the influence of night visibility to the definition of safe stopping sight distance is crucial. It was determined that the overall resulting shorter sight distances during nighttime driving could potentially lead to unsafe driving conditions. The authors suggested that design policies should be reconsidered in order to address the issue of safe stopping sight distance at nighttime.

In the development of ISA's, there is the need to identify the potential of the system to adhere to expected deceleration rates as well as stopping distances. It must be stated, however, that, in the development of these models, their validation could only be realized using field operational tests. In particular, for most of the ISA systems developed and tested using simulator experiments, existing or potential deceleration rate models and stopping distance equation validation have not been addressed.

\section{Overview of AVSAS and other ISA features}

In order to adapt to different driving styles, driver speed behaviour was analyzed and classified in the absence of any ISA system. The purpose was to develop a profile for classification or clustering of drivers in order to be able to develop an adaptable ISA to the individual's speeding and braking preferences. As shown in Figure 1, a clustering algorithm was applied to driver's behaviour as recorded in the first phase of simulator experiment. During this first phase of the experiment, subjects drove the simulator without any ISA system active. Drivers' average and maximum acceleration, deceleration, and speeds on different roadway segments were analyzed, and resulted in three clusters of drivers, with drivers classified as conservative, normal, or aggressive. Based on this analysis, values for excess speed and maximum deceleration rates were chosen for each of these groups of drivers.

The values produced by the clustering algorithm provide input for the main routine in the AVSAS system, which runs continuously while the vehicle is in 


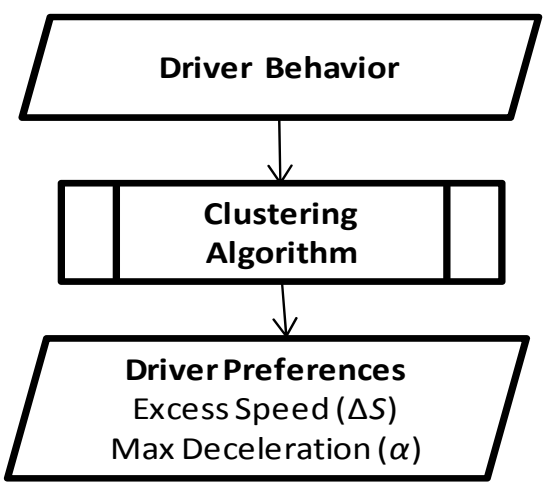

Figure 1: Driver behaviour clustering.

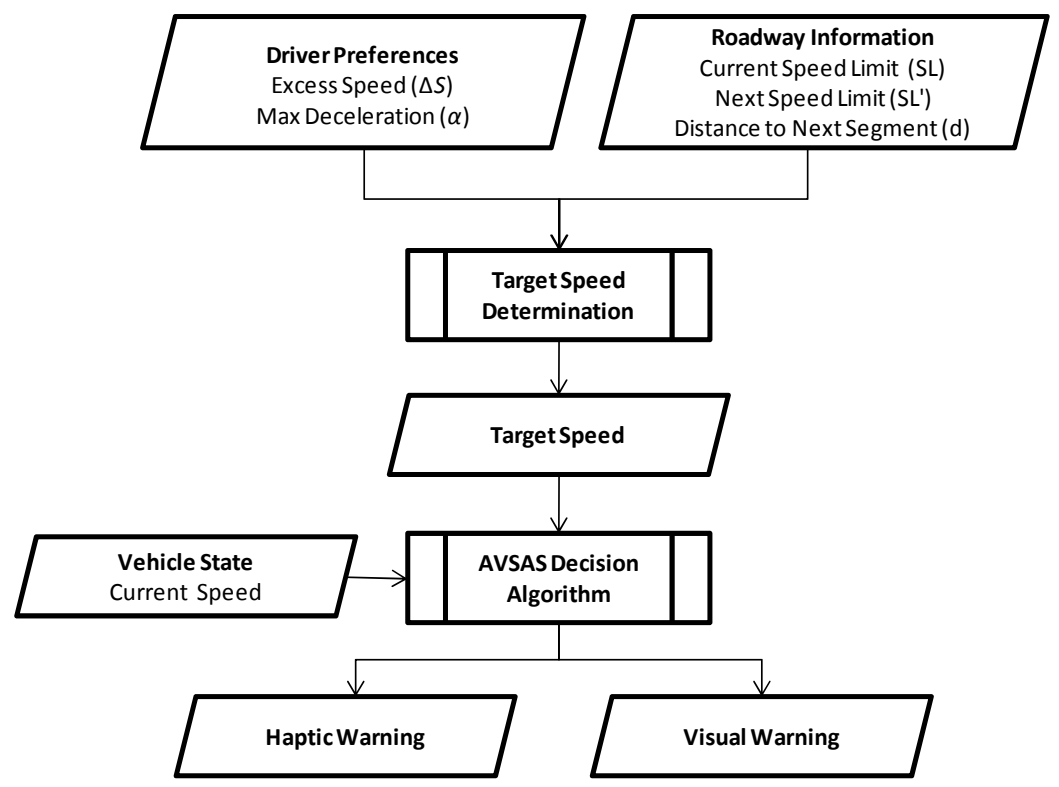

Figure 2: $\quad$ AVSAS speed regulation process.

motion. This process, shown in Figure 2, begins with a determination of the current target speed based on driver preferences and the current roadway information.

The following equation is used for the Target Speed (TS) determination:

$$
\mathrm{TS}=\min \left\{S L+\Delta S \sqrt{ }\left\{\left(S L^{\prime}+\Delta S\right)^{2}+2 \alpha d\right\}\right\}
$$

where $\quad T S$ is the target speed in meters/second,

$S L$ is the speed limit for the current segment of roadway in meters/second,

$\Delta S$ is the driver's preferred excess speed in meters/second, 
$S L^{\prime}$ is the speed limit on the next segment of roadway in meters/second,

$\alpha$ is the driver's preferred deceleration rate $(\alpha>0)$, in meters $/$ second $^{2}$, and

$d$ is the distance between the vehicle and the beginning of the next segment of roadway in meters.

Note that this determination of target speed allows AVSAS to adapt to individual speed profiles, driving scenario and roadway geometry and condition. When drivers approach potential scenarios that require a reduction in speed such as intersections, work zones and curves, AVSAS takes into consideration of the drivers' preferred deceleration rates to determine the target speed at the given moment. AVSAS considers the speed limit for curves to be equal to the posted advisory speed. For special cases that require a complete stop, e.g. a red light or stop sign, AVSAS considers the Speed Limit and the preferred excess speed to be equal to 0 , guiding the driver to a stop in these situations. The calculated Target Speed guides the presentation of system generated warnings. The details of the warning presentation are described in the next section.

\section{Methodology}

Speed data for twenty-one drivers were obtained for the each of the ISAs introduced using a driving simulator software program, STISM. The three ISAs are the 1) Warning, 2) Mandatory and 3) AVSAS. AVSAS was developed by the Centre for Intelligent System Research at George Washington University to improve on the efficacy of the Warning ISA as well as the acceptance of the Mandatory ISA [14]. The Warning ISA provides alarms and notifications (voice or visual) when the system determines that a driver has exceeded the speed limit or advisory. The advisory systems provide information to the driver such as the current recommended speed based on the prevailing road and weather conditions. The Mandatory ISA, which is an automatic control system, prevents the driver from exceeding a prevailing speed limit by using mechanical systems and techniques such as throttle control and braking. The Mandatory ISA, while effective in ensuring compliance with speed limits, is viewed extremely negatively by drivers. On the other hand, the Warning ISA system is significantly less effective in reducing driver speeds $[14,15]$ AVSAS functions as a speed management system based on individual driver speeding preferences under different roadway scenarios rather than a complete speed limiting system. AVSAS allows a driver to exceed the prevailing speed limit up to a certain threshold beyond which it functions as a Mandatory ISA, with override capability. It also has all features of a Warning ISA including advance hazard warning displays.

The driving simulator experiment involved six (6) types of road classes. These are freeway $(65 \mathrm{mph})$, collector $(45 \mathrm{mph})$, local $(35 \mathrm{mph})$, residential (25 $\mathrm{mph})$, rural $(55 \mathrm{mph})$, and urban roads $(35 \mathrm{mph})$. The driving scenarios included 5 curves $(20,35,45$ and $55 \mathrm{mph}$ advisory speed limits), 3 stop-controlled intersections, a fog zone, and an accident zone. 
The regression models were developed based on the data gathered on 21 drivers using the three ISAs. The ISA development and evaluation data, as mentioned earlier, included several other scenarios. The portions of the data on various intersections (for stopping distance at stop-controlled intersections) and curves (for approach speed on curves) were used to develop the regression models. The models were developed using multiple regression analysis, with all models indicators tested at $5 \%$ level of significance. The significance and adequacies of these models were examined using a number of statistical indicators and tests including the F-test, $t$-test, the two-sample KolmogorovSmirnov test (KS test), associated $p$-values, homoscedasticity and normality.

\section{Results}

\subsection{Stopping distance models at stop-sign controlled intersection}

The multiple linear regression technique was used to develop a statistical model to assess the relationship between the approach speed, maximum deceleration rate, stopping distance and the deceleration time. These values were obtained for each driver on approach to the intersection. In the database, the instant when the brake was applied on approach to the intersection was noted by the value of the "Brake Pedal Force" as recorded in the STISM software program.

The form of the model was:

where

$$
\mathrm{D}=\alpha_{0}+\alpha_{1} a+\alpha_{2} V+\alpha_{3} t
$$

$\mathrm{D}=$ distance to stop sign when braking is initiated ( $\mathrm{ft})$,

$a=$ maximum deceleration rate $\left(\mathrm{ft} / \mathrm{s}^{2}\right)$,

$V=$ approach speed $(\mathrm{ft} / \mathrm{s})$, and

$t=$ deceleration time (s).

The constants $\alpha_{0}, \alpha_{1}, \alpha_{2}$ and $\alpha_{3}$ are the coefficients of the regression model with an associated error of $\varepsilon\left[\varepsilon \sim N\left(0, \sigma^{2}\right)\right]$. The resulting regression models developed for the Warning, Mandatory and AVSAS intelligent speed adaptation systems are presented in Table 1.

The above models passed the tests for normality and homoscedasticity while the KS tests (depicted in Figure 3) showed that there is no statistically significant

Table 1: $\quad$ Summary of regression models for stopping distance at stop signs.

\begin{tabular}{|l|l|c|c|c|}
\hline \multicolumn{1}{|c|}{ ISA Type } & \multicolumn{1}{|c|}{ Model } & $\mathbf{R}^{2}$ & $\boldsymbol{F}_{[2,18]}$ & $\begin{array}{c}\text { Model } \\
\boldsymbol{p} \text {-Value }\end{array}$ \\
\hline Warning & $\begin{array}{l}\mathrm{D}=103.6-13.75 a+4.304 \\
6.909 t\end{array}$ & 0.91 & 54.90 & $<0.0001$ \\
\hline Mandatory & $\mathrm{D}=46.23-8.21 a+3.126 V+8.602 t$ & 0.88 & 37.78 & $<0.0001$ \\
\hline AVSAS & $\begin{array}{l}\mathrm{D}=37.66-13.56 a+4.844 V+ \\
11.94 t\end{array}$ & 0.87 & 35.20 & $<0.0001$ \\
& \multicolumn{1}{|c|}{} & & & \\
\hline
\end{tabular}




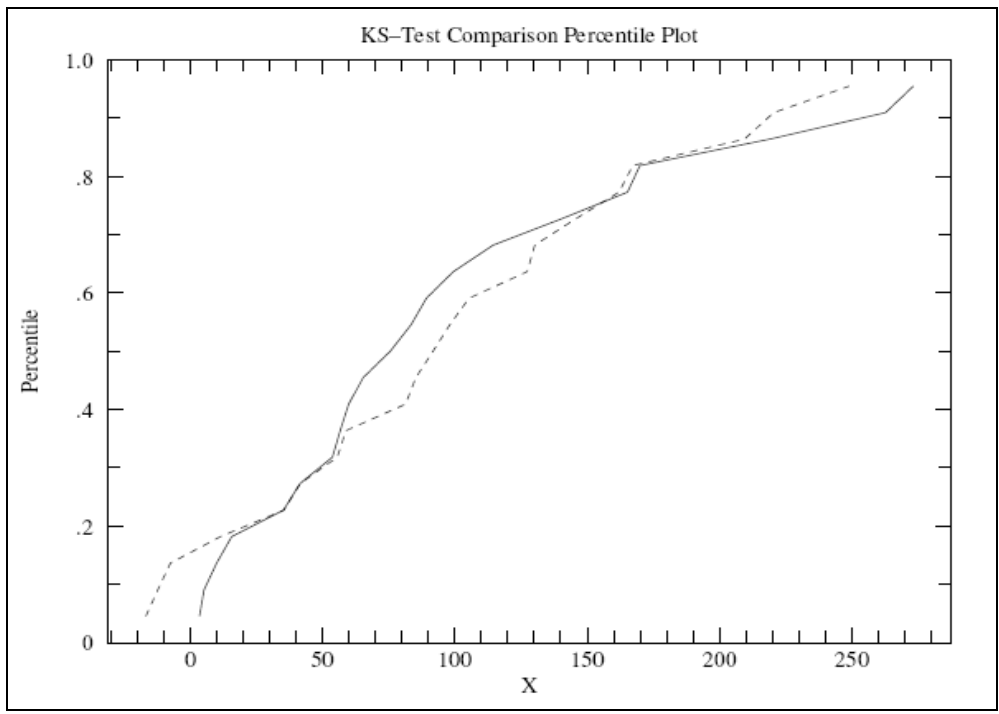

3(a) Warning ISA

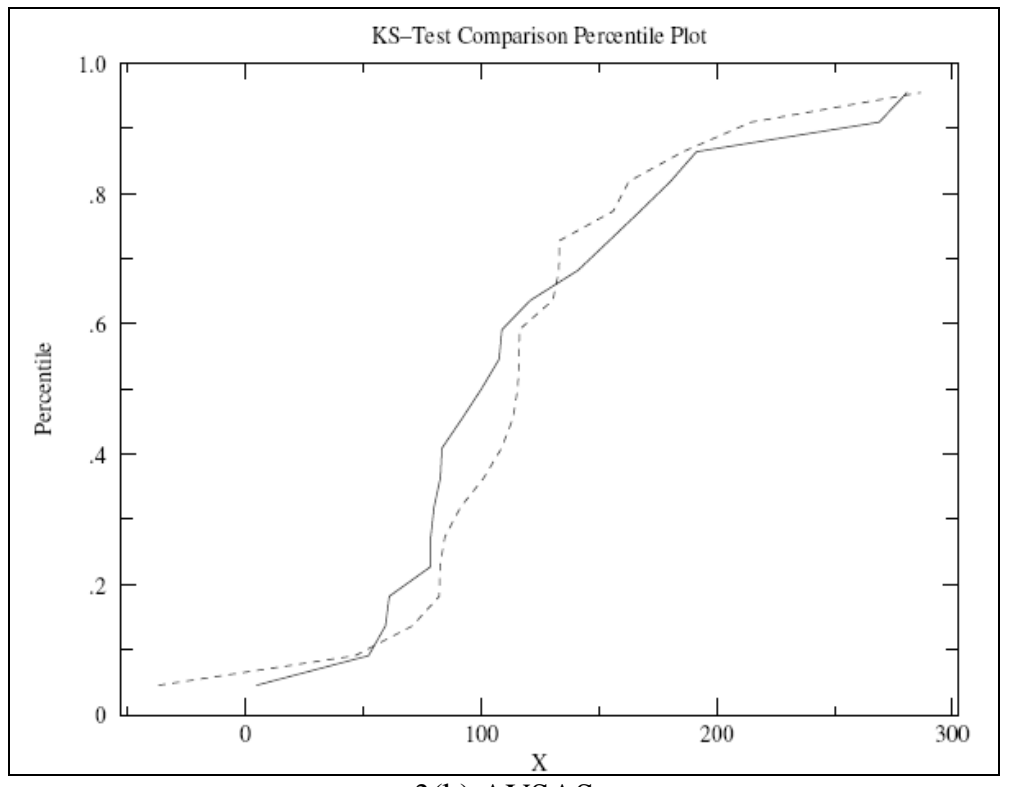

3(b) AVSAS

Figure 3: Plots for two-sample Kolmogorov-Smirnov tests. 


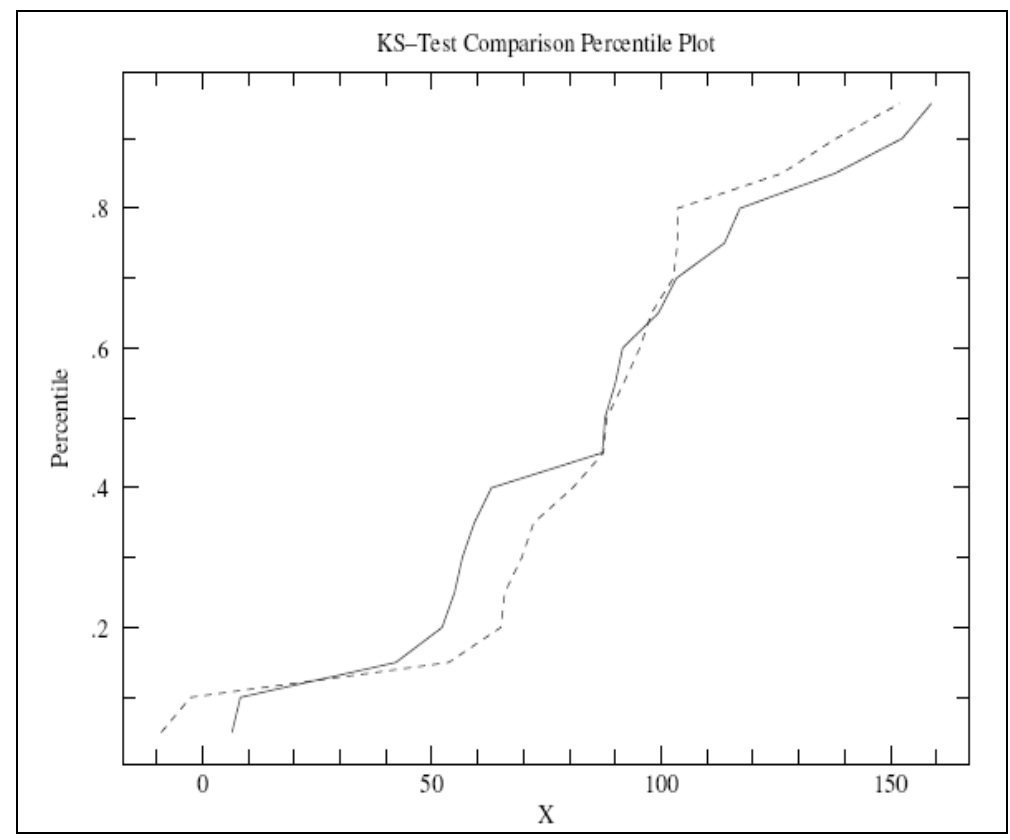

3(c) Mandatory ISA

Figure 3: Continued.

difference between the predicted and observed values, at 5\% level of significance. The dotted lines in Figure 3 ((a), (b) and (c)) represents the model's output.

From the regression analysis of these stopping distance regression models for each of the ISA types, the approach speed, maximum deceleration rate and deceleration time adequately predicted the stopping distance for all the drivers on approach to the stop-controlled intersection, with $p<0.05$ for the $t$-statistics for the corresponding coefficients.

\subsection{Deceleration rate model on curve approach}

Regression models were also developed for the driver' deceleration rate on approach to a curve with a radius of 489 feet used in the simulator. The curve had a posted speed limit of $35 \mathrm{mph}$. The multiple linear regression technique was used to develop a statistical model to assess the relationship between the approach speed, maximum deceleration rate, and the deceleration time. These values were obtained for each driver on approach to the curve. The model assumed is of the form:

$$
\sqrt{V}=\beta_{0}+\beta_{1} a+\beta_{2} t^{2}
$$

where

$$
\begin{aligned}
& a=\text { maximum deceleration rate }\left(\mathrm{ft} / \mathrm{s}^{2}\right), \\
& V=\operatorname{approach} \text { speed }(\mathrm{ft} / \mathrm{s}), \text { and } \\
& t=\text { deceleration time }(\mathrm{s}) .
\end{aligned}
$$


The constants $\beta_{0}, \beta_{1}$, and $\beta_{2}$ are the coefficients of the regression model with an associated error of $\varepsilon\left[\varepsilon \sim N\left(0, \sigma^{2}\right)\right]$. The resulting predicting models for the ISAs are presented in Table 2 .

Table 2: $\quad$ Summary of regression models for simulation-based deceleration rates at curves.

\begin{tabular}{|l|c|c|c|c|}
\hline \multicolumn{1}{|c|}{ ISA Type } & Model & $\mathbf{R}^{2}$ & $\boldsymbol{F}_{[2,18]}$ & $\begin{array}{c}\text { Model } \\
\boldsymbol{p} \text {-Value }\end{array}$ \\
\hline Warning & $\sqrt{V}=7.994+0.0636 a-0.0532 t^{2}$ & 0.66 & 17.54 & $<0.0001$ \\
\hline Mandatory & $\sqrt{V}=9.249-0.0016 a-0.5492 t^{2}$ & 0.90 & 82.08 & $<0.0001$ \\
\hline AVSAS & $\sqrt{V}=10.02+0.015 a-0.77 t^{2}$ & 0.93 & 112.37 & $<0.0001$ \\
\hline
\end{tabular}

The deceleration rate could be obtained by taking the first derivative of the approach speed, $V$. These models passed the tests for normality and homoscedasticity while the KS tests (as presented in Figure 4 (a), (b) and (c)) showed that there is no statistically significant difference between the predicted and observed values, at $5 \%$ level of significance. The associated $p$-values for the $t$-statistics for the coefficients were less than 0.05 , indicating that the approach speed in the regression models on approach to the curve for the three ISAs were adequately predicted by the maximum deceleration rate and the deceleration time.

\section{Conclusions}

The driver simulator models presented in this paper could be subsequently incorporated into the design of the road segments for the applicable scenarios for future ISA tests. This could help determine the metrics that are needed in the design process of the ISA systems which would realistically address the behaviour of drivers using a driving simulator. From the analysis of the stopping distance regression models for each of the ISA scenarios, the approach speed, maximum deceleration rate and deceleration time adequately predicted the stopping distance for all the drivers on approach to the stop-controlled intersection in a driving simulator. The coefficient of determination for all the models $\left(R^{2}\right)$ range from $87 \%$ to $91 \%$ which gives an indication that the independent variables, all taken together, adequately explain the variations in the dependent variable. Similarly, the regression models for the drivers on approach to the curve for the three ISAs show that the approach speed can be adequately predicted by the maximum deceleration rate and the deceleration time. The $R^{2}$ values range from $66 \%$ to $93 \%$. The KS tests conducted also affirms the notion that the regression models developed for the various ISA treatments are adequate. 


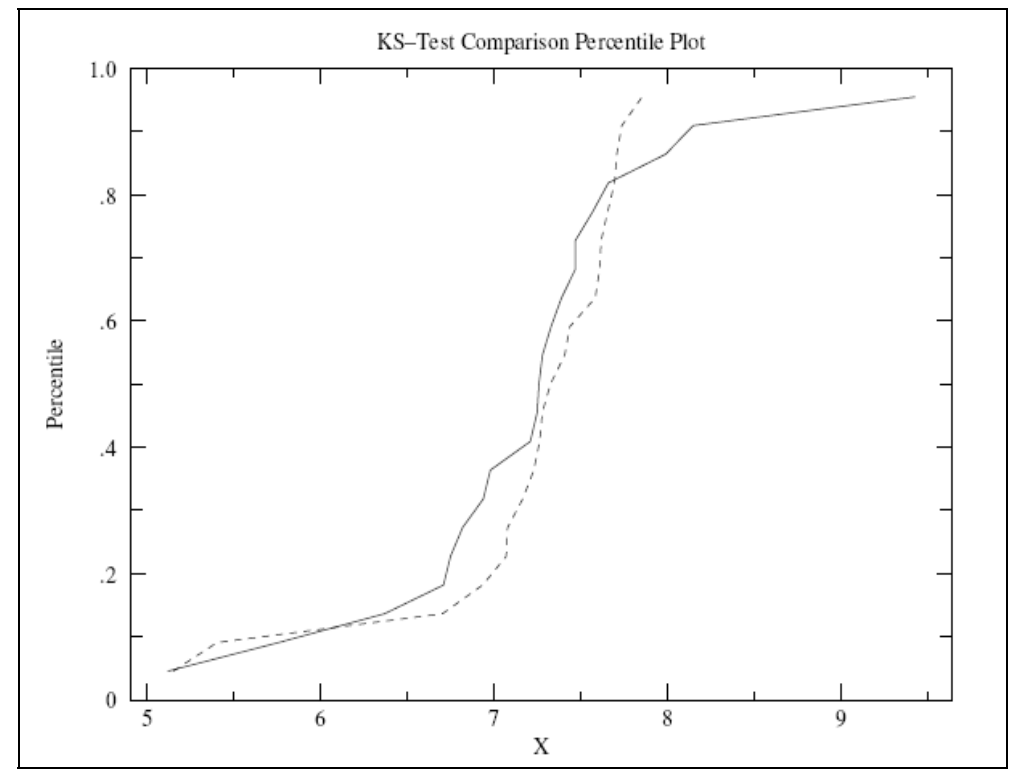

4(a) Warning ISA

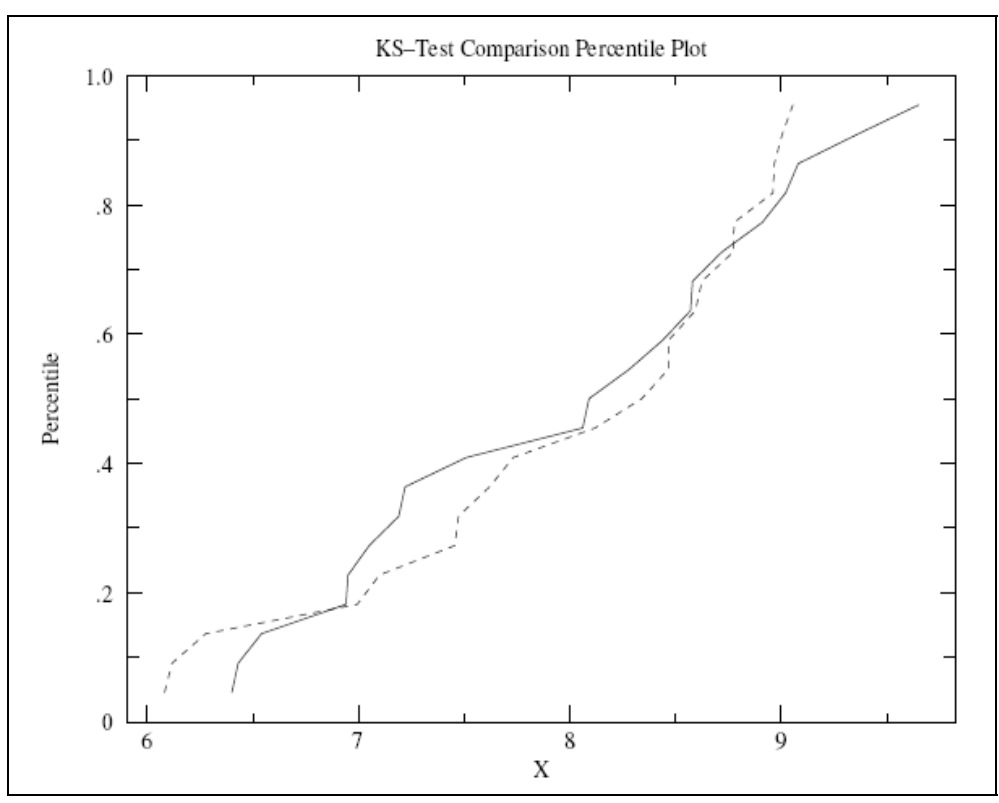

4(b) AVSAS

Figure 4: $\quad$ Plots for two-sample Kolmogorov-Smirnov tests. 


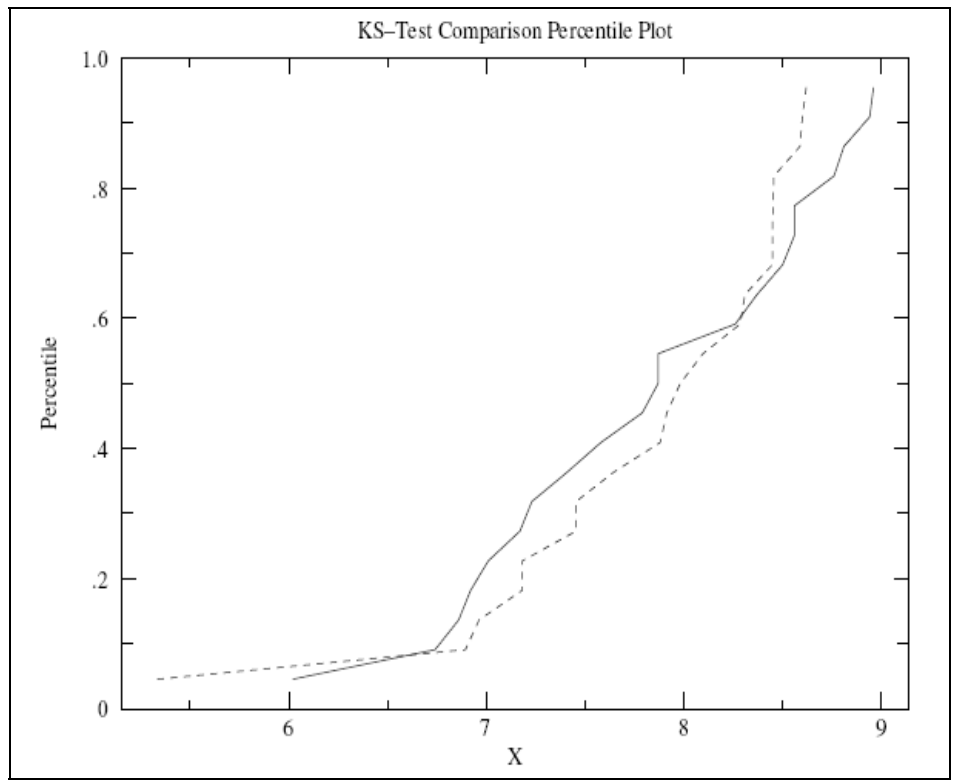

4(c) Mandatory ISA

Figure 4: Continued.

\section{Acknowledgement}

Appreciation is extended to the Turner-Fairbank Highway Research Centre, Highway Research Division, for supporting this study.

\section{References}

[1] Akcelik and Besley, aaSIDRA 2.0 User Guide and Manual, Akcelik and Associates, ARRB Transport Research Ltd., Victoria, Australia, 2002.

[2] Gazis, D., Herman R., and Maradudin A,. The Problem of the Amber Light Traffic Flow. Operations Research, 8(1), 1960.

[3] Parsonson, P.S. and Santiago, A., Design Standards for Timing the TrafficSignal Clearance Period Must be Improved to Avoid Liability. Compendium of Technical Papers, Institute of Transportation Engineers 50th Annual Meeting, Pittsburgh, PA, 1980.

[4] Wortman, R. H., and Matthias, J. S., An evaluation of driver behaviour at signalized intersections. Arizona Transportation and Traffic Institute, Tucson, AZ, 1983.

[5] Institute of Transportation Engineers, Traffic Engineering Handbook, $5^{\text {th }}$ ed., Prentice Hall, Saddle River, NJ, 1999.

[6] Wortman, R.H., Fox, T.C., An Evaluation of Vehicle Deceleration Profiles. Journal of Advanced Transportation, 28 (3), 1994. 
[7] Samuels, S.E., Jarvis, J., Acceleration of and Deceleration of Modern Vehicles, Australian Road Research Report 86, Australian Research Board, Victoria, Australia, 1978.

[8] Bennett, C.R. and Dunn, R.C.M., Driver Deceleration Behaviour on a Freeway in New Zealand. Transportation Research Record, 1510, pp. 70$75,1995$.

[9] Crisman B., Perco, P., Robba, A., Roberti, R., Deceleration Model for TwoLane Rural Roads, Advances in Transportation Studies, 11, pp. 19-32, 2007.

[10] Haas, R., Inman V., Dixson A., and Warren, D., Use of Intelligent Transportation Systems Data to Determine Driver Deceleration and Acceleration Behaviour. Transportation Research Record, 1899, pp. 3-10, 2004.

[11] American Association of State Highway and Transportation Officials (AASHTO), Policy on Geometric Design of Highways and Streets, $4^{\text {th }}$ Edition, Washington, DC, Chap. 3, 109-113, 2001.

[12] Fambro, D.B., Fitzpatrick, K. and Koppa, R.J., New Stopping Sight Distance Model for use in Highway Design, Transportation Research Record, 1701, pp. 1-8, 2000.

[13] Apostolopoulou, D., Psarianos, B., Stamatiadis, N., and Kasapi, E., Investigation of Stopping Sight Distances Validity during Nighttime, Road and Transportation Research Association, FGSV 002/67, pp. 400-409, 2000.

[14] Sayed R.A., Delaigue, P., Blum, J., Mortazavi, A., Eskandarian, A., Development of an Intelligent Multimode Speed Adaptation System. SAE 01-1321, 2007.

[15] Arhin, S., Blum, J., Eskandarian, A., Delaigue, P., and Soudbakhsh, D., "Effectiveness and Acceptance of Adaptive Intelligent Speed Adaptation Systems", Transportation Research Record (TRR), Journal of the Transportation Research Board, , Intelligent Transportation Systems and Vehicle Highway Automation, No. 2086, pp. 133-139, 2008, 